\title{
EDITORIAL
}

\section{IN THE FEBRUARY 2009 ISSUE OF CLINICS}

\author{
Mauricio Rocha-e-Silva, Editor
}

doi: $10.1590 / \mathrm{S} 1807-59322009000200001$

In this issue of CLINICS we are publishing the first report on organ transplants from Hospital das Clínicas, covering the period of 2002 to 2007 . We intend to follow up this article with annual reports starting early 2010. In this article Azeka et al. report on a total of 3,321 solid organ and tissue transplants at the institution. The 5-year survival curve ranged from $53 \%$ to $88 \%$, and shows that solid organ and tissue transplants are feasible within the institution, and leads one to expect that the quality of transplantants should improve over the coming years.

We are also publishing an Editorial commenting on a review article published in our January 2009 issue, eight more original clinical science articles, and one basic research article, as well as 3 case reports.

Salluh et al. describe outcomes and thrombotic events in a series of 18 critically ill cancer patients positive for antiphospholipid antibodies. Measurements were performed up to 48 hours after the occurrence of acrocyanosis or arterial/ venous thrombotic events. All patients developed MOF during the ICU stay, and intra-hospital death occurred in 13/18 ( $72 \%$ ) of the patients. Of the 5 discharged patients, 3 were alive 90 days later. Authors conclude that the coexistence of sepsis or SIRS and aPL antibodies was often associated with MOF and death.

Furtado et al. developed and validated a BrazilianPortuguese version of the Gesture Behavior Test for patients with non-specific chronic low back pain. Translation into Portuguese was performed by a rheumatologist fluent in the language of origin (French), and skilled in the validation of questionnaires. The translated version was back-translated into French by a native-speaking teacher of the language. The two translators then created a final consensual version in Portuguese. They conclude that the Brazilian version proved to be a reproducible and valid instrument. In addition, according to the questionnaire results, more disabled patients

Hospital das Clínicas, Faculdade de Medicina da Universidade de São Paulo - São Paulo/SP, Brazil.

mrsilva36@hcnet.usp.br were found to exhibit more protective gesture behavior related to low-back.

Ruano et al. report on their experience using selective fetoscopic laser photocoagulation of superficial placental anastomoses for the treatment of 19 monochorionic diamniotic twin pregnancies complicated by severe twin-twin transfusion syndrome. A $1.0 \mathrm{~mm}$ fetoscopic laser was employed for this therapy and showed results similar to those reported in the literature for larger endoscopes.

Greve et al. compared radial shockwaves and conventional physiotherapy for treating plantar fasciitis in 32 patients equally divided between conventional treatment (10 physiotherapy sessions each, consisting of ultrasound, kinesiotherapy and instruction for stretching exercises at home) and a test group (undergoing 3 applications of radial shockwaves, once a week, plus instruction for stretching exercises at home). They found that both treatments were effective for pain reduction and for improving the functional abilities of patients with plantar fasciitis, but that the effect of the shockwaves appeared sooner than physiotherapy after the onset of treatment.

Torquato et al. quantified the interaction between intraabdominal pressure and positive-end expiratory pressure in 30 mechanically ventilated ICU patients with a fixed tidal volume; respiratory system plateau and abdominal pressure were measured at a Positive-End Expiratory Pressure level of zero and $10 \mathrm{~cm} \mathrm{H}_{2} \mathrm{O}$, with and without a $5 \mathrm{~kg}$ weight placed over their stomachs. They conclude that the addition of the weight onto the abdomen increased both abdominal pressure and airway plateau pressure, confirming that intra-abdominal hypertension elevates the plateau pressure. However, they add, plateau pressure should not be taken as a good detector of elevated intra-abdominal pressure in patients under mechanical ventilation using PEEP, adding that intra-abdominal pressure must also be measured.

Bacarin et al. investigated and compared the influence of a previous history of foot ulcers on plantar pressure variables during gait of patients with diabetic neuropathy. The study included 20 controls; 17 diabetic neuropathy patients 
without foot ulcers, and 10 diabetic neuropathy patients with at least one healed foot ulcer within the last year, and concludes that foot ulcers in the clinical history of diabetic neuropathy influenced plantar pressure distribution, resulting in an increased load under the midfoot and rearfoot and an increase in the variability of plantar pressure during barefoot gait, whereas the progression of diabetic neuropathy was not found to influence plantar pressure distribution.

Lopes et al. compared self-report and interview administration methods of the Western Ontario Rotator Cuff Index and the Disabilities of Arm, Shoulder and Hand Questionnaires in 30 male and female patients with rotator cuff disorders in 30 male and female patients over 18 years of age with rotator cuff disorders (tendinopathy or rotator cuff tear) and Brazilian Portuguese as their primary language. The majority $(n=26)$ had a complete college degree or higher. Mean questionnaire scores and administration times were not significantly different $(\mathrm{p}<0.05)$ between the two groups procedures. There were statistically significant correlations $(\mathrm{p}<0.05)$ between the 2 procedures and strong correlations were found between the questionnaires in both groups, with no differences between them with regard to administration time or correlations between the 2 questionnaires.

Duarte et al. measured the prevalence of thyroid dysfunction in 399 elderly cardiology patients with mild excessive iodine intake in the urban area of São Paulo. They found that elderly patients have a higher prevalence of both hypo- and hyperthyroidism as well as thyroid nodules when compared with the general population; about one-third of the older patients had elevated urinary secretion of iodine and a higher prevalence of chronic Hashimoto's thyroiditis. Authors recommend that ultrasonographic studies, tests for thyroid function and autoimmunity should be performed in elderly patients.

Ýrkörüicii et al. analyzed the formation of adherences in 50 Wistar male rats undergoing median laparotomy and standardized abrasion of the visceral and parietal peritoneum, Phosphatidylcholine, Seprafilm II, and t-PA alone and phosphatidylcholine and t-PA in combination were tested. Seven days after surgery, a relaparotomy for adhesion grading showed that adhesion was less pronounced in the study groups, even more so in the combination group. In the t-PA group and the combination group, six and two rats, respectively, developed hematomas locally on the cecum. 